\title{
Accessibility and availability of health care services to internally displaced persons, in Kitgum and Pader districts, northern Uganda
}

\author{
Christopher Garimoi Orach ${ }^{1^{*}}$, Juliet Faith Aporomon $^{2}$, Nelson Musoba $^{3}$, Lukwiya Micheal $^{4}$ \\ ${ }^{1}$ Department of Community Health and Behavioural Sciences, Makerere University School of Public Health, Kampala, Uganda; \\ *Corresponding Author: cgorach@musph.ac.ug \\ ${ }^{2}$ Uganda Bureau of Statistics (UBOS), Kampala, Uganda; aporo_ju@yahoo.com \\ ${ }^{3}$ Ministry of Health (MoH), Kampala, Uganda; nmusoba@yahoo.com \\ ${ }^{4}$ World Health Organisation (WHO), Kampala, Uganda; mlukwiya@who.afro.int
}

Received 6 May 2013; revised 6 June 2013; accepted 1 July 2013

Copyright (C) 2013 Christopher Garimoi Orach et al. This is an open access article distributed under the Creative Commons Attribution License, which permits unrestricted use, distribution, and reproduction in any medium, provided the original work is properly cited.

\section{ABSTRACT}

Introduction: During 1986 - 2009, an estimated 1.6 million persons were internally displaced from northern and eastern regions of Uganda due to civil war. We investigated accessibility to and availability of health care services for the internally displaced persons in Kitgum and Pader districts, northern Uganda. Methods: This was a cross-sectional study conducted in Kitgum and Pader districts, northern Uganda. We interviewed a total of 1383 respondents comprising 968 (70\%) adults and $415(30 \%)$ adolescents; $60 \%$ were females and $40 \%$ males, randomly selected from 35 of $67(52.2 \%)$ internally displaced persons (IDP) camps. We held 27 key informant interviews and $\mathbf{5 2}$ focus group discussions. Data were entered in EPI data version 3.02 and analysed using SPSS version 12.00 statistical packages. Findings: Two thirds of the respondents $67.5 \%$ lived within $5 \mathrm{~km}$ distance of a health facility. The majority $62.9 \%$ of respondents mentioned that health related information was readily provided. $43.5 \%$ of health providers were always available in a health facility. A quarter $\mathbf{2 5 . 1 \%}$ of health facilities always had drugs available, while $56.9 \%$ of the drugs prescribed were always available. Two thirds of the respondents $65.9 \%$ were satisfied with the health care services provided. The main reasons for the choice of a health facility were proximity $29.6 \%$, provision of free treatment $24.7 \%$ and availability of drugs $17.2 \%$. Main barriers to health care access were due to the lack of financial resources, train- ed personnel, and inadequate drugs and supplies in the health facilities. Conclusions and Recommendations: The majority of IDPs lived in close proximity to health facilities and obtained health care services from public health facilities. Access to health care was determined mainly by proximity and availability of free services and drugs. Although geographic accessibility to health services was high, lack of finances, information and decision power hindered access to health care services.

Keywords: Accessibility; Availability; Health Care Services; Quality; Satisfaction; Internally Displaced Persons; Uganda

\section{INTRODUCTION}

Over two decades of a protracted civil war between the Lord's Resistance Army (LRA) rebels and the government armed forces, led to the displacement of an estimated 1.6 million persons from northern and eastern regions of Uganda during 1986-2009 [1]. Most of the displaced persons originated from the Acholi sub-region, in the districts of Gulu, Lamwo, Amuru, Kitgum, Nwoya and Pader, that bore the brunt of the war.

In 2005 , the vast majority $95 \%$ or 279,000 and $93 \%$ or 267,000 of the populations of Pader and Kitgum districts respectively were displaced into squalid internally displaced persons camps. A total of 64 internally displaced persons camps were established, 30 in Kitgum and 34 in Pader districts respectively [2].

During the peak period of displacement in 2005-2006, 
the health indices in the Acholi sub-region were poor with high crude and under five mortality rates, estimated at 1.54 and 3.18 per 10,000/day respectively. The crude mortality rate (CMR) and the under five mortality rate (U5MR) in Gulu district IDP camps were high, estimated at 1.22 and 2.31 per 10,000/day respectively. These mortality rates were both above the emergency thresholds of $1 / 10,000 /$ day and $2 / 10,000$ /day respectively and the national estimates of $0.46 / 10,000 /$ day and $0.98 / 10,000 /$ day respectively [3].

During emergencies, displaced populations especially women and children are extremely vulnerable to ill health owing to a variety of socio-cultural, economic and physiologic reasons [4,5]. Several factors including insecurity, poverty, poor nutrition, inadequate water and sanitation, and lack of adequate health services predispose displaced populations to high levels of morbidity and mortality [4]. Excess mortality in displaced population settings is often caused by the same diseases that affect the non displaced populations including acute lower respiratory tract infection (ARI), malaria, measles, diarrhoeal diseases and malnutrition $[4,6]$.

In situations of emergencies the health system is heavily burdened, and the problems of the quality of services and inadvertent discrimination may arise, yet it should ensure respect for human rights and the attainment of the highest standard of health [7]. Displaced populations including IDPs and refugees face various challenges in accessing health care due to socio-economic, geographic, cultural factors [8]. The un-availability of health services means that displaced population living in under serviced areas, may not obtain appropriate services. A study by Reitz (1995) highlights the need to bring health resources and care centres to areas where marginalized populations are highly concentrated [9].

Since 2007, the phenomenon of displacement has gradually evolved into return home (resettlement) and the camp population shifted into decongestion settlements in northern Uganda. Today the majority, over $90 \%$, of the population in the Acholi sub-region has returned home. The objective of this study was to examine accessibility to and availability of health care services to internally displaced persons (IDPs) in Kitgum and Pader war affected districts, northern Uganda.

\section{METHODS}

\subsection{Study Sites}

The study was carried out in Kitgum and Pader districts in northern Uganda. In 2007, there were an estimated 629,617 internally displaced persons i.e. 310,111 IDPs in Kitgum and 319,506 in Pader districts respectively [10]. The districts had a total of 67 IDP camps i.e. 24 camps in Kitgum and 43 in Pader district respectively.
Figure 1 shows the location of the study district in northern Uganda.

\subsection{Study Design}

We carried a cross-sectional study comprising internally displaced community members including adults and adolescents, service providers at the central and local district levels (including Ministry of Health and local district health services providers and humanitarian relief i.e. UN agencies, international NGOs and community based organisations (CBOs).

\subsection{Data Collection Procedures}

We sampled 15 of 24 IDP camps (62.5\%) in Kitgum district and 20 of 43 (46.5\%) camps in Pader using both purposive and random sampling techniques. The camps were stratified by parishes, population size, and security considerations and randomly selected into the study.

Administratively, a camp is sub-divided into blocks and zones. A block constituted a study cluster. We selected households based on the modified WHO Expanded Programme of Immunization cluster sampling technique [11]. The centre of the cluster was identified. A bottle was spun to determine the initial direction of movement. A random starting household was identified by listing all households from the centre to the end of the cluster and a random starting household was chosen, and the next selected for interview was the nearest to the one where the interviewed had been conducted.

In a household, either a male or female adult head of household was interviewed. We selected male or female respondents in the proportion of $40 \%$ to $60 \%$ respectively. However, after every third household, an adolescent aged between 14 and 19 years was identified and interviewed. A total of 51 respondents (adult/adolescent) were interviewed per cluster in Kitgum and 40 in Pader district. In total 1383 individuals comprising 720 (52\%) of respondents in Pader and 663 (48\%) in Kitgum district were interviewed.

We held 27 in-depth interviews and conducted 52 focus group discussions. The key informants included camp commandants, and Local Council I, II and III Chairpersons, in charge of health units including health centre II and III. In addition, leaders of community-based organisations (CBOs) were interviewed. At the district level, civic authorities including local council (LC V) chairpersons, resident district commissioners, chief administrative officers; and in the health sector, the district health team members such as the district health officer (DHO), medical superintendent, medical officers, the person in charge of the nurse training school (the matron), district health visitor, and personnel responsible for health centre IV were interviewed. Finally, we interviewed various 


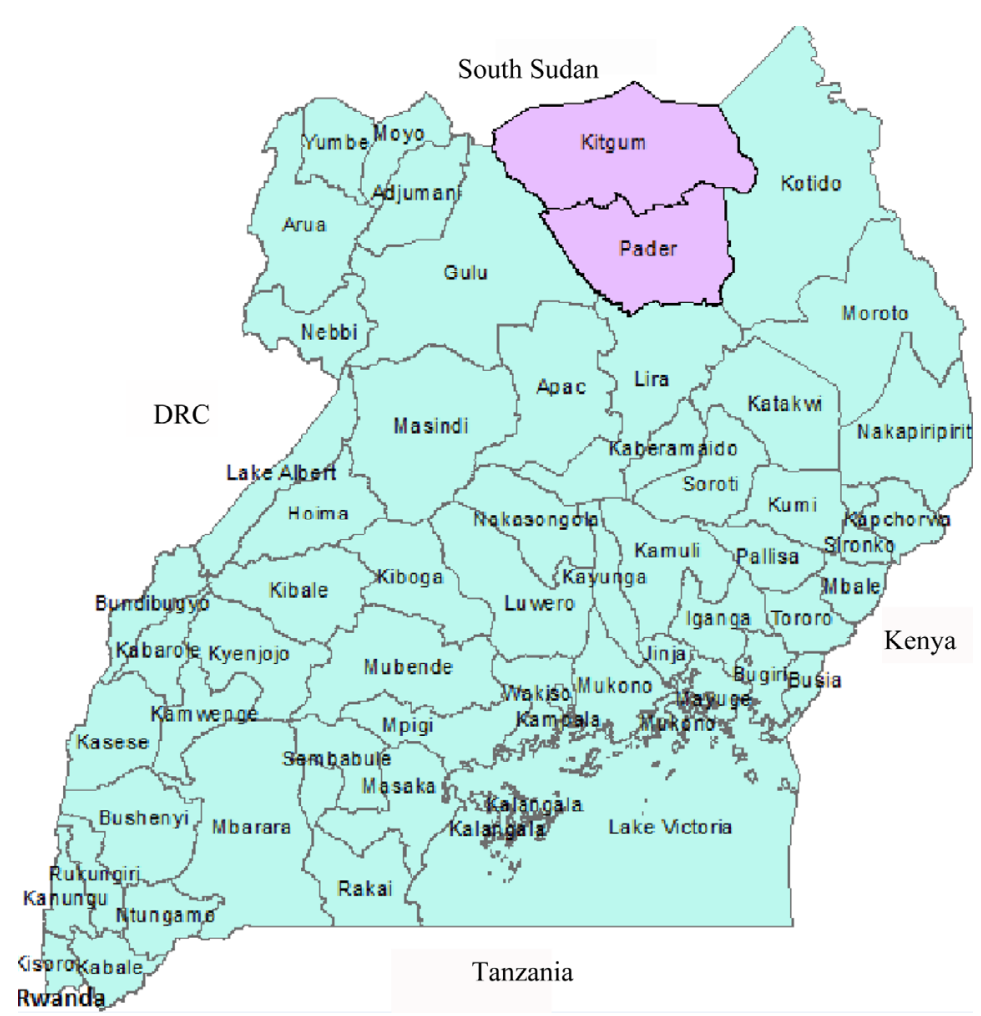

Figure 1. Map showing study districts, Kitgum and Pader, northern Uganda.

humanitarian relief workers from agencies including United Nations High Commission for Human Rights (UNHCHR), United Nations Children's Fund (UNICEF), World Health Organization (WHO), and Non-Governmental Organisations as shown (in Table 1).

We held 27 in-depth key informant interviews with 14 staff from international and local humanitarian agencies (UN/NGO/CBOs), 8 with district health staff, and 5 with community and district civic leaders.

We conducted a total of 52 focus group discussions (FGDs) (Table 2). The FGDs consisted of adult-men and women, adolescent, and community leader groups. Women and men were divided in both adult and adolescent FGDs. There were eight to ten persons per group. The discussions took about one hour each. The focus group discussions were taped recorded.

More than half of the FGDs (28 of 52, or 52.8\%), were carried out with adults (men and women), 16 of 52 (30.8\%) with adolescents (males and females), and 7 of 52 (13.5\%) with community leaders.

Data collectors (research assistants) were trained for three days. The study instruments comprising (questionnaire, focus group, and key informant guides) were translated into the local language-Luo/Acholi and back into English. The data collection tools were pre-tested. Quantitative data were captured, cleaned, and edited. The completed questionnaires were checked by the principal investigator daily for accuracy, completeness and consis- tency before the data collectors left the IDP study sites/ camps. Quantitative data were checked for completeness, sorted, coded and captured using EPI data version 3.02 packages. The data were entered in Epidata version 3.1 and analyzed using SPSS version 12.00 software package. Qualitative data were analyzed manually. Content analysis was based on condensation and abstraction of main themes.

\section{RESULTS}

As shown in Table 3, more than a quarter of the respondents $26.1 \%$ (25.7\% males and $26.4 \%$ females) were below 19 years of age. The majority of adult respondents $61.4 \%$ ( $57.3 \%$ males and $64.2 \%$ females) were in the age group 20 - 49 years. $24.2 \%$ of respondents $(9.1 \%$ males and a significantly higher proportion of females, 37.4\%) had no formal education while $56.8 \%$ of respondents had attained at least primary level education. Most respondents $75.2 \%$ (73.9\% males and 76.4\% females) have lived in encampments for a period ranging between one and ten years.

Table 4 shows that most (78\%) respondents (79.5\% in Kitgum and 76.5\% in Pader) live within $5 \mathrm{~km}$ of a health facility. The main reasons for choice of a health facility are proximity to health facility $29.6 \%$ (males $29.1 \%$ and females $30.1 \%$ ), availability of free treatment $22.7 \%$ (males $21.5 \%$ and females $23.9 \%$ ); availability of drugs 
Table 1. Key informants interviewed.

\begin{tabular}{ccc}
\hline Category & Number & Percentage (\%) \\
\hline Health provider & 8 & 29.6 \\
Community/Civic leaders & 5 & 18.5 \\
UN/NGOs/CBOs & 14 & 51.9 \\
Total & $\mathbf{2 7}$ & $\mathbf{1 0 0}$ \\
\hline
\end{tabular}

Keys: $\mathrm{UN}=$ United Nations, $\mathrm{NGO}=$ Non governmental organization, $\mathrm{CBO}=$ Community based organization .

Table 2. Categories of focus group discussions conducted by district.

\begin{tabular}{ccccc}
\hline Category & Kitgum & District & & \\
\hline & $\mathbf{n}$ & Pader & Total & N \\
Adults & & $\mathbf{n}$ & & 26.9 \\
Women & 9 & 5 & 14 & 26.9 \\
men & 7 & 7 & 14 & 13.5 \\
Community Leaders & 3 & 4 & 7 & 13.5 \\
Adolescent & & & 7 & 17.3 \\
Males & 4 & 4 & 9 & $\mathbf{1 0 0}$ \\
Females & 5 & 4 & $\mathbf{5 2}$ & \\
Total & $\mathbf{2 8}$ & $\mathbf{2 4}$ & & \\
\hline
\end{tabular}

Table 3. Respondents' socio-demographic characteristics and duration of encampment.

\begin{tabular}{|c|c|c|c|c|c|c|}
\hline & \multicolumn{6}{|c|}{ Sex } \\
\hline & \multicolumn{2}{|c|}{ Male } & \multicolumn{2}{|c|}{ Female } & \multicolumn{2}{|c|}{ Total } \\
\hline & $\mathrm{n}$ & $(\%)$ & $\mathrm{n}$ & (\%) & $\mathrm{n}$ & (\%) \\
\hline \multicolumn{7}{|l|}{ Age groups in years } \\
\hline$<19$ & 141 & $(25.7)$ & 210 & $(26.4)$ & 351 & (26.1) \\
\hline $20-29$ & 114 & $(20.8)$ & 226 & $(28.4)$ & 340 & (25.3) \\
\hline $30-39$ & 124 & $(22.6)$ & 182 & $(22.9)$ & 306 & (22.8) \\
\hline $40-49$ & 76 & (13.9) & 103 & $(12.9)$ & 179 & (13.3) \\
\hline $50-59$ & 54 & (9.9) & 39 & $(4.9)$ & 93 & (6.9) \\
\hline $60-69$ & 24 & $(4.4)$ & 31 & (3.9) & 55 & (4.1) \\
\hline $70+$ & 15 & $(2.7)$ & 5 & $(0.6)$ & 20 & $(1.5)$ \\
\hline Total & 548 & $(100)$ & 796 & $(100)$ & 1344 & (100) \\
\hline \multicolumn{7}{|l|}{ Education status } \\
\hline No formal education & 50 & (9.1) & 300 & $(37.4)$ & 350 & $(26.0)$ \\
\hline Primary & 342 & $(62.3)$ & 426 & $(53.1)$ & 768 & (56.8) \\
\hline Secondary & 138 & $(25.1)$ & 65 & (8.1) & 203 & $(15.0)$ \\
\hline Tertiary & 19 & (3.5) & 8 & $(1.0)$ & 27 & (2.0) \\
\hline Other & 0 & $(0.0)$ & 3 & $(0.4)$ & 3 & $(0.2)$ \\
\hline Total & 549 & $(100)$ & 802 & $(100)$ & 1351 & (100) \\
\hline \multicolumn{7}{|l|}{ Duration of encampment } \\
\hline$<1$ year & 77 & $(14.0)$ & 114 & $(15.6)$ & 191 & $(14.9)$ \\
\hline $1-5$ years & 279 & $(50.8)$ & 388 & $(53.2)$ & 667 & $(52.2)$ \\
\hline $6-10$ years & 127 & $(23.1)$ & 160 & $(21.9)$ & 287 & (22.4) \\
\hline 11 - 15 years & 36 & (6.6) & 31 & $(4.2)$ & 67 & $(5.2)$ \\
\hline $16-20$ years & 19 & (3.5) & 24 & (3.3) & 43 & $(3.4)$ \\
\hline$>20$ years & 11 & $(2.0)$ & 13 & (1.8) & 24 & $(1.9)$ \\
\hline Total & 549 & (100) & 730 & (100) & 1279 & (100) \\
\hline
\end{tabular}


Table 4. Access to health care services and information.

\begin{tabular}{|c|c|c|c|c|c|c|c|c|c|c|c|c|}
\hline \multicolumn{13}{|c|}{ District } \\
\hline & \multicolumn{4}{|c|}{ Kitgum } & \multicolumn{4}{|c|}{ Pader } & \multicolumn{4}{|c|}{ Total } \\
\hline & \multicolumn{2}{|c|}{ Male } & \multicolumn{2}{|c|}{ Female } & \multicolumn{2}{|c|}{ Male } & \multicolumn{2}{|c|}{ Female } & \multicolumn{2}{|c|}{ Male } & \multicolumn{2}{|c|}{ Female } \\
\hline & $\mathrm{n}$ & $(\%)$ & $\mathrm{n}$ & $(\%)$ & $\mathrm{n}$ & $(\%)$ & $\mathrm{n}$ & $(\%)$ & $\mathrm{n}$ & $(\%)$ & $\mathrm{n}$ & $(\%)$ \\
\hline \multicolumn{13}{|l|}{ Distance to health facility } \\
\hline $0-5 \mathrm{~km}$ & 200 & $(76.3)$ & 306 & $(82.7)$ & 218 & $(76.8)$ & 324 & $(76.2)$ & 418 & $(76.6)$ & 630 & $(79.2)$ \\
\hline $6-10 \mathrm{~km}$ & 40 & $(15.3)$ & 42 & $(11.4)$ & 55 & $(19.4)$ & 88 & $(20.7)$ & 95 & $(17.4)$ & 130 & $(16.4)$ \\
\hline $11-15 \mathrm{~km}$ & 15 & (5.7) & 11 & (2.9) & 4 & (1.4) & 6 & (1.4) & 19 & (3.5) & 17 & $(2.1)$ \\
\hline $16-20 \mathrm{~km}$ & 6 & (2.3) & 10 & $(2.7)$ & 2 & $(0.7)$ & 1 & $(0.3)$ & 8 & (1.5) & 11 & (1.4) \\
\hline$>20 \mathrm{~km}$ & 1 & $(0.4)$ & 1 & $(0.3)$ & 5 & $(1.7)$ & 6 & (1.4) & 6 & (1) & 7 & $(0.9)$ \\
\hline Total & 262 & (100) & 370 & (100) & 284 & (100) & 425 & (100) & 546 & $(\mathbf{1 0 0 )}$ & 795 & (100) \\
\hline \multicolumn{13}{|c|}{ Ownership of health facility } \\
\hline Public facility & 201 & $(76.7)$ & 284 & $(76.6)$ & 174 & $(60.8)$ & 290 & $(67.3)$ & 375 & $(68.4)$ & 574 & $(71.6)$ \\
\hline PNFP facility & 23 & (8.8) & 37 & (10) & 52 & $(18.2)$ & 73 & $(17)$ & 75 & $(13.7)$ & 110 & $(13.7)$ \\
\hline Private & 32 & $(12.2)$ & 35 & $(9.4)$ & 25 & $(8.7)$ & 31 & $(7.2)$ & 57 & $(10.4)$ & 66 & $(8.2)$ \\
\hline Traditional & 1 & $(0.4)$ & 9 & (2.4) & 22 & (7.7) & 29 & (6.7) & 23 & $(4.2)$ & 38 & $(4.7)$ \\
\hline CORPs/Village & 2 & $(0.8)$ & 2 & $(0.5)$ & 2 & $(0.7)$ & 4 & $(0.9)$ & 4 & $(0.7)$ & 6 & $(0.8)$ \\
\hline Other & 3 & $(1.1)$ & 4 & $(1.1)$ & 11 & $(3.9)$ & 4 & $(0.9)$ & 14 & (2.6) & 8 & (1) \\
\hline Total & 262 & (100) & 371 & (100) & 286 & (100) & 431 & $(100)$ & 548 & $(\mathbf{1 0 0})$ & 802 & (100) \\
\hline \multicolumn{13}{|c|}{ Reason for choice of health facility } \\
\hline Facility is near & 123 & $(27.4)$ & 186 & $(29.7)$ & 160 & $(31.5)$ & 236 & $(30.4)$ & 283 & $(29.1)$ & 422 & $(30.1)$ \\
\hline $\begin{array}{l}\text { Provision of free } \\
\text { treatment }\end{array}$ & 93 & $(20.7)$ & 145 & (23.1) & 117 & (23.1) & 191 & $(24.6)$ & 210 & $(21.5)$ & 336 & (23.9) \\
\hline Availability of drugs & 86 & $(19.2)$ & 125 & (19.9) & 84 & $(16.6)$ & 114 & $(14.7)$ & 170 & $(17.4)$ & 239 & (17) \\
\hline Good quality services & 56 & $(12.5)$ & 67 & $(10.7)$ & 44 & (8.7) & 79 & $(10.1)$ & 120 & $(12.3)$ & 146 & $(10.4)$ \\
\hline $\begin{array}{l}\text { Provide advanced } \\
\text { services }\end{array}$ & 28 & $(6.2)$ & 32 & (5.1) & 28 & (5.5) & 42 & (5.4) & 56 & (5.7) & 74 & (5.3) \\
\hline Skilled health worker & 25 & (5.6) & 26 & $(4.1)$ & 22 & (4.3) & 30 & (3.9) & 47 & (4.8) & 56 & (4) \\
\hline Payment for condoms & 24 & (5.3) & 35 & (5.6) & 22 & $(4.3)$ & 33 & $(4.2)$ & 46 & $(4.7)$ & 68 & (4.8) \\
\hline Staff polite & 12 & $(2.7)$ & 6 & (1) & 17 & (3.4) & 39 & (5) & 29 & (3) & 45 & $(3.2)$ \\
\hline Trust/confidentiality & 2 & $(0.4)$ & 5 & $(0.8)$ & 13 & (2.6) & 13 & $(1.7)$ & 15 & $(1.5)$ & 18 & (1.3) \\
\hline Total & 449 & (100) & 627 & (100) & 507 & (100) & 777 & (100) & 976 & $(100)$ & 1404 & (100) \\
\hline \multicolumn{13}{|l|}{$\begin{array}{l}\text { Provision of health related } \\
\text { information }\end{array}$} \\
\hline Readily & 185 & $(71.1)$ & 223 & $(61.6)$ & 170 & $(62.7)$ & 232 & $(56.6)$ & 355 & $(66.8)$ & 455 & (59) \\
\hline Sometimes & 53 & (20.4) & 89 & $(24.6)$ & 67 & $(24.7)$ & 112 & $(27.3)$ & 120 & $(22.6)$ & 201 & (26) \\
\hline With difficulty & 13 & (5) & 36 & (9.9) & 24 & (8.9) & 39 & (9.5) & 37 & (7) & 75 & (9.7) \\
\hline Never & 9 & (3.5) & 14 & (3.9) & 10 & (3.7) & 27 & (6.6) & 19 & (3.6) & 41 & (5.3) \\
\hline Total & 260 & (100) & 362 & $(\mathbf{1 0 0 )}$ & 271 & $(\mathbf{1 0 0 )}$ & 410 & (100) & 531 & $(\mathbf{1 0 0 )}$ & 772 & (100) \\
\hline \multicolumn{13}{|c|}{ Disclosure of cause of illness } \\
\hline Yes & 167 & $(64.2)$ & 187 & $(50.7)$ & 162 & $(56.8)$ & 204 & $(47.4)$ & 329 & $(60.4)$ & 391 & $(48.9)$ \\
\hline No & 93 & $(35.8)$ & 179 & $(48.5)$ & 117 & $(41.1)$ & 221 & $(51.4)$ & 210 & $(38.5)$ & 400 & $(50.1)$ \\
\hline Don’t know & 0 & $(0)$ & 3 & $(0.8)$ & 6 & (2.1) & 5 & $(1.2)$ & 6 & (1.1) & 8 & (1) \\
\hline Total & 260 & (100) & 369 & $(\mathbf{1 0 0})$ & 285 & (100) & 430 & (100) & 545 & $(\mathbf{1 0 0 )}$ & 799 & (100) \\
\hline
\end{tabular}

Keys PNFP = private not for profit; CORPS = community owned resource persons. 
$17.2 \%$ (17.4\% males and females $17.0 \%)$ and good quality services $11.4 \%$ (12.3\% males and females $10.4 \%)$. The majority $62.9 \%$ (66.8\% males and 59\%) mentioned that health related information is readily available and $54.7 \%$ (60.4\% males and $48.9 \%$ females) mentioned that causes of illness is disclosed by health providers.

As shown in Table 5, a quarter $25.1 \%$ of respondents (22.8\% males and $27.3 \%$ females) mentioned that drugs are always available; while $56.9 \%$ (62.2\% males and $51.5 \%$ females) mentioned that prescribed drugs are all available and $43.5 \%$ ( $41.5 \%$ males and $45.5 \%$ females) reported that health service providers are always available in the facilities.

Table 6 revealed that the majority of respondents $56.1 \%$ (47.1\% males and a significantly higher proportion of females $65.0 \%$ ) mentioned that it takes a very long time to see a provider. The main constraints faced in accessing health care include lack of money $36.4 \%$ (34.9\% male and reported by a higher proportion 37.9\% female); lack of information $24.3 \%$ (25.3\% males and $23.2 \%$ females) and lack of decision power men tioned by $17.5 \%$ ( $17.7 \%$ males and $17.3 \%$ females).

As shown in Table 7, the majority of respondents $79.0 \%$ (77.7\% males and $80.3 \%$ females) consider consultation rooms private; similarly $81.0 \%$ (80.2\% males and $81.8 \%$ females considered the information provided are confidentially handled by providers. The majority $68.5 \%$ (71.0\% males and $65.9 \%$ females) were at least satisfied or highly satisfied with the health care services provided.

\section{Barriers to Health Care Access}

Several barriers to health care accessibility were identified. These included long distance to health facility, poor attitude of health care workers (being rude), inadequate staffing, lack of drugs and supplies in the health facilities as well as high costs of health care services. The costs of health care access were reported to be high and un-affordable to many community members. Hence some people have resorted to using traditional medication which they consider cheaper.

“... some of the expectant mothers are referred to $\mathrm{Ka}$

Table 5. Availability of drugs, supplies and personnel in health facility.

\begin{tabular}{|c|c|c|c|c|c|c|c|c|c|c|c|c|}
\hline \multicolumn{13}{|c|}{ District } \\
\hline & \multicolumn{4}{|c|}{ Kitgum } & \multicolumn{4}{|c|}{ Pader } & \multicolumn{4}{|c|}{ Total } \\
\hline & \multicolumn{2}{|c|}{ Male } & \multicolumn{2}{|c|}{ Female } & \multicolumn{2}{|c|}{ Male } & \multicolumn{2}{|c|}{ Female } & \multicolumn{2}{|c|}{ Male } & \multicolumn{2}{|c|}{ Female } \\
\hline & $\mathrm{n}$ & (\%) & $\mathrm{n}$ & (\%) & $\mathrm{n}$ & (\%) & $\mathrm{n}$ & (\%) & $\mathrm{n}$ & (\%) & $\mathrm{n}$ & (\%) \\
\hline \multicolumn{13}{|c|}{$\begin{array}{c}\text { Availability of drugs in } \\
\text { health facility }\end{array}$} \\
\hline Always & 52 & $(20.1)$ & 83 & $(22.5)$ & 71 & (25.3) & 134 & (31.5) & 123 & $(22.8)$ & 217 & (27.3) \\
\hline Sometimes & 164 & (63.3) & 222 & $(60.1)$ & 151 & (53.7) & 201 & $(47.3)$ & 315 & $(58.3)$ & 423 & (53.3) \\
\hline Rarely & 39 & $(15.1)$ & 53 & $(14.4)$ & 56 & (19.9) & 76 & (17.9) & 95 & $(17.6)$ & 129 & $(16.2)$ \\
\hline Never & 4 & $(1.5)$ & 11 & (3) & 3 & (1.1) & 14 & (3.3) & 7 & (1.3) & 25 & (3.2) \\
\hline Total & 259 & (100) & 369 & (100) & 281 & $(\mathbf{1 0 0})$ & 425 & (100) & 540 & (100) & 794 & (100) \\
\hline \multicolumn{13}{|c|}{$\begin{array}{c}\text { Availability of drugs } \\
\text { prescribed }\end{array}$} \\
\hline All available & 149 & $(60.3)$ & 160 & $(46.5)$ & 172 & (63.9) & 224 & (55.9) & 321 & $(62.2)$ & 384 & (51.5) \\
\hline Some available & 87 & $(35.2)$ & 162 & $(47.1)$ & 85 & (31.6) & 154 & $(38.4)$ & 172 & (33.3) & 316 & $(42.4)$ \\
\hline Not available & 11 & $(4.5)$ & 22 & (6.4) & 12 & $(4.5)$ & 23 & $(5.7)$ & 23 & (4.5) & 45 & (6.1) \\
\hline Total & 247 & (100) & 344 & (100) & 269 & (100) & 401 & (100) & 516 & $(100)$ & 745 & (100) \\
\hline \multicolumn{13}{|c|}{$\begin{array}{l}\text { Availability of condoms } \\
\text { in health facility }\end{array}$} \\
\hline Yes & 186 & (71.3) & 258 & (69.9) & 209 & (72.8) & 298 & $(69.4)$ & 395 & $(72.1)$ & 556 & (69.7) \\
\hline No & 57 & (21.8) & 74 & (20.1) & 59 & (20.6) & 84 & (19.6) & 116 & $(21.2)$ & 158 & (19.8) \\
\hline Don't know & 18 & (6.9) & 37 & (10) & 19 & (6.6) & 47 & (11) & 37 & (6.7) & 84 & (10.5) \\
\hline Total & 261 & (100) & 369 & (100) & 287 & (100) & 429 & (100) & 548 & (100) & 798 & (100) \\
\hline \multicolumn{13}{|c|}{$\begin{array}{l}\text { Availability of personnel } \\
\text { in health facility }\end{array}$} \\
\hline Always & 81 & (31) & 140 & (37.9) & 145 & $(51.2)$ & 221 & (52) & 226 & $(41.5)$ & 361 & (45.5) \\
\hline Sometimes & 147 & (56.3) & 180 & (48.8) & 99 & (35) & 146 & (34.4) & 246 & $(45.2)$ & 326 & (41.1) \\
\hline Rarely & 31 & (11.9) & 44 & (11.9) & 36 & (12.7) & 51 & (12) & 67 & (12.3) & 95 & (11.9) \\
\hline Never & 2 & $(0.8)$ & 5 & (1.4) & 3 & (1.1) & 7 & $(1.6)$ & 5 & (1) & 12 & (1.5) \\
\hline Total & 261 & (100) & 369 & (100) & 283 & (100) & 425 & (100) & 544 & $(100)$ & 794 & (100) \\
\hline
\end{tabular}


Table 6. Barriers to health care accessibility.

\begin{tabular}{|c|c|c|c|c|c|c|c|c|c|c|c|c|}
\hline \multicolumn{13}{|c|}{ District } \\
\hline & \multicolumn{4}{|c|}{ Kitgum } & \multicolumn{4}{|c|}{ Pader } & \multicolumn{4}{|c|}{ Total } \\
\hline & \multicolumn{2}{|c|}{ Male } & \multicolumn{2}{|c|}{ Female } & \multicolumn{2}{|c|}{ Male } & \multicolumn{2}{|c|}{ Female } & \multicolumn{2}{|c|}{ Male } & \multicolumn{2}{|c|}{ Female } \\
\hline & $\mathrm{n}$ & $(\%)$ & $\mathrm{n}$ & $(\%)$ & $\mathrm{n}$ & $(\%)$ & $\mathrm{n}$ & $(\%)$ & $\mathrm{n}$ & $(\%)$ & $\mathrm{n}$ & $(\%)$ \\
\hline \multicolumn{13}{|l|}{ Time taken to see a provider } \\
\hline Very long $>2$ hrs & 125 & $(48.3)$ & 242 & $(65.8)$ & 131 & $(46.1)$ & 274 & $(64.3)$ & 256 & $(47.1)$ & 516 & $(65)$ \\
\hline Long 1 - 2 hours & 62 & $(23.9)$ & 68 & $(18.5)$ & 69 & $(24.3)$ & 71 & $(16.7)$ & 131 & $(24.1)$ & 139 & (17.5) \\
\hline Not long $<1$ hour & 34 & $(13.1)$ & 28 & $(7.6)$ & 42 & $(14.8)$ & 48 & $(11.3)$ & 76 & $(14.1)$ & 76 & (9.6) \\
\hline Didn’t wait & 38 & $(14.7)$ & 30 & $(8.1)$ & 42 & $(14.8)$ & 33 & $(7.7)$ & 80 & $(14.7)$ & 63 & $(7.9)$ \\
\hline Total & 259 & (100) & 368 & (100) & 284 & (100) & 426 & (100) & 543 & $(100)$ & 794 & (100) \\
\hline \multicolumn{13}{|l|}{$\begin{array}{c}\text { Constraints in accessing } \\
\text { health services }\end{array}$} \\
\hline Lack of money & 70 & $(29.4)$ & 137 & $(37.2)$ & 123 & (39) & 198 & (38.4) & 193 & (34.9) & 335 & (37.9) \\
\hline Lack of information & 60 & $(25.2)$ & 74 & $(20.1)$ & 80 & $(25.4)$ & 131 & $(25.4)$ & 140 & $(25.3)$ & 205 & $(23.2)$ \\
\hline Lack of decision power & 53 & $(22.3)$ & 74 & $(20.1)$ & 45 & (14.3) & 79 & $(15.3)$ & 98 & $(17.7)$ & 153 & (17.3) \\
\hline Other & 55 & $(23.1)$ & 83 & $(22.6)$ & 67 & $(21.3)$ & 108 & (20.9) & 122 & $(22.1)$ & 191 & (21.6) \\
\hline Total & 238 & (100) & 368 & (100) & 315 & (100) & 516 & (100) & 553 & $(100)$ & 884 & (100) \\
\hline \multicolumn{13}{|l|}{$\begin{array}{c}\text { Dialogue on medication } \\
\text { prescribed }\end{array}$} \\
\hline Yes & 54 & (27) & 51 & $(17.5)$ & 61 & $(29.7)$ & 91 & $(30.7)$ & 115 & $(28.4)$ & 142 & $(24.2)$ \\
\hline No & 144 & (72) & 236 & (81.1) & 133 & (64.9) & 197 & (66.6) & 277 & $(68.4)$ & 433 & (73.8) \\
\hline Don’t know & 2 & (1) & 4 & $(1.4)$ & 11 & $(5.4)$ & 8 & $(2.7)$ & 13 & $(3.2)$ & 12 & (2) \\
\hline Total & 200 & $(100)$ & 291 & $(\mathbf{1 0 0 )}$ & 205 & $(100)$ & 296 & $(100)$ & 405 & $(100)$ & 587 & (100) \\
\hline
\end{tabular}

Table 7. Perceptions about privacy, confidentiality and satisfaction with the quality of health care services.

\begin{tabular}{|c|c|c|c|c|c|c|c|c|c|c|c|c|}
\hline \multicolumn{13}{|c|}{ District } \\
\hline & \multicolumn{4}{|c|}{ Kitgum } & \multicolumn{4}{|c|}{ Pader } & \multicolumn{4}{|c|}{ Total } \\
\hline & Male & & Female & & Male & & Female & & Male & & Female & \\
\hline & $\mathrm{n}$ & $(\%)$ & $\mathrm{n}$ & $(\%)$ & $\mathrm{n}$ & $(\%)$ & $\mathrm{n}$ & $(\%)$ & $\mathrm{n}$ & $(\%)$ & $\mathrm{n}$ & (\%) \\
\hline \multicolumn{13}{|l|}{$\begin{array}{c}\text { Privacy of consultation } \\
\text { rooms }\end{array}$} \\
\hline Yes & 192 & (73.8) & 281 & (76.4) & 232 & $(81.1)$ & 360 & $(83.7)$ & 424 & $(77.7)$ & 641 & $(80.3)$ \\
\hline No & 68 & (26.2) & 87 & (23.6) & 54 & (18.9) & 70 & $(16.3)$ & 122 & (22.3) & 157 & (19.7) \\
\hline Total & 260 & (100) & 368 & (100) & 286 & (100) & 430 & (100) & 546 & (100) & 798 & (100) \\
\hline \multicolumn{13}{|l|}{ Confidentiality } \\
\hline Yes & 201 & (77.3) & 290 & (78.6) & 236 & (82.8) & 363 & (84.6) & 437 & $(80.2)$ & 653 & (81.8) \\
\hline No & 40 & (15.4) & 47 & (12.7) & 34 & (11.9) & 53 & $(12.4)$ & 74 & (13.6) & 100 & (12.6) \\
\hline Don’t know & 19 & (7.3) & 32 & $(8.7)$ & 15 & $(5.3)$ & 13 & (3) & 34 & $(6.2)$ & 45 & (5.6) \\
\hline Total & 260 & (100) & 369 & (100) & 285 & $(100)$ & 429 & (100) & 545 & $(100)$ & 798 & (100) \\
\hline \multicolumn{13}{|c|}{$\begin{array}{l}\text { Satisfaction with health } \\
\text { care services }\end{array}$} \\
\hline Highly satisfied & 61 & (23.5) & 65 & (17.5) & 57 & (20) & 59 & $(13.8)$ & 118 & (21.6) & 124 & $(15.5)$ \\
\hline Satisfied & 129 & (49.6) & 195 & (52.6) & 141 & $(49.1)$ & 208 & $(48.6)$ & 270 & $(49.4)$ & 403 & $(50.4)$ \\
\hline Marginally satisfied & 35 & (13.4) & 65 & $(17.5)$ & 44 & (15.3) & 71 & $(16.6)$ & 79 & $(14.4)$ & 136 & (17) \\
\hline Not satisfied & 34 & (13.1) & 43 & (11.6) & 42 & $(14.6)$ & 86 & $(20.1)$ & 76 & (13.9) & 129 & $(16.1)$ \\
\hline Very unsatisfied & 1 & $(0.4)$ & 3 & $(0.8)$ & 3 & (1) & 4 & $(0.9)$ & 4 & $(0.7)$ & 7 & (1) \\
\hline Total & 260 & (100) & 371 & (100) & 287 & (100) & 428 & (100) & 547 & (100) & 799 & (100) \\
\hline
\end{tabular}

longo hospital which is very far and expensive to reach in terms of transport costs...”. Adult Women, Lukole.

"Some people have resorted to using traditional herbs that cure ailments so they feel no need to visit health facilities”. FGD Adolescent Youth, Padibbe East.
Several key informants and focus groups mentioned that most people prefer to seek care first in government health centres because the services are free. However, several barriers exist in accessing services in these government health facilities. These barriers include; 
- Generally late opening and early closure of the health facilities. Opening hours generally range from 10.00 am to $1.00 \mathrm{pm}$.

- Few health workers. Sometimes there are only 2 personnel in a facility. Moreover, some the health workers were rude and uncooperative towards patients. The poor attitude and rudeness of some health providers was mentioned by several groups;

"The health personnel are rude and uncooperative towards patients.” KII, Pader District.

“... some health providers abuse patients..." Adult Women, Lukole.

Lack of drugs and supplies was mentioned as a constraint in Kitgum public hospital, compared to Kalongo faith based facility-hospital. Moreover it was mentioned that the quality of service is better in Kalongo where the services are provided quickly;

"We choose to go to health centre III because of lack of money to go to the hospital and because of the availability of free drugs in the health centres" Adolescent Girls, Corner Kilak.

The majority (64.9\%) of the female respondents mentioned that waiting time is very long in the health facilities. Both males and females attribute the long waiting time to the presence of many patients.

"After waiting for a long time, the health workers always tell us "Come tomorrow" So many people often go to get treatment in other health facilities-government or private clinic. Male Youth FGD, Paloga Camp.

"people go for testing in the government health centres and then go for help in other centres like the Meeting Point for help”. Male Youth FGD. Paloga Camp, Kitgum District.

Although the majority $79.0 \%$ (77.7\% of males and $80.3 \%$ females), consider the consultation room as private in the settings, the respondents were concerned about lack of visual and auditory privacy in the facilities as highlighted in the excerpts below;

"There is no visual privacy at the health centre, when a person is inside with the doctor, he/she can be seen by other patients, one sits on the form with others and speaks out his problem" and everything they discuss can be heard... everything is done in one room". Female Adults Obolokome Camp; Male adult FGD, Palabek Kal.

"Some health workers gossip a lot, they do not keep secrets. Many people do not like them". Adolescent Youth Padibe East.

\section{DISCUSSION}

This study showed that several factors influence access to health care services including proximity to health facility, provision of free treatment services and availability of drugs and supplies in the war torn northern di- stricts of Uganda. Our findings are similar with other studies which identified several socio-economic and geographic factors that determine access to health care [8, 12]. We found that the majority of the displaced population had access to a health facility within $5 \mathrm{~km}$ distance in the two war affected districts. The main sources of health care were government (public) health facilities. In the war affected northern districts, like in the rest of the country, most primary health care facilities are public. Nationwide $59 \%$ of the hospitals and $78 \%$ of the lower health facilities are public [13]. Therefore, strategies to improve the capacity of existing public health facilities, at both first line and referral, in such emergency settings are critical. This implies the need to enhance accessibility of health care services through establishing a functional referral system for health emergencies (including medical, surgical and obstetric care); ensuring availability of human resources for health, especially skilled attendants at birth; as well as ensuring supply of essential drugs and supplies. The support of humanitarian organizations including the United Nations agencies e.g. WHO, and international NGOs to the local health system to carry out effective health care services is critical. Previous studies by Orach and De Brouwere (2006) showed that in similarly affected conflict settings of the West Nile region, Uganda improved access to health care led to better met obstetric needs of refugees than for the host population [12].

The study revealed that the majority of clients readily received health related information from the health facilities. Access to health information is a right [14] and vital towards guiding community decision making on health matters. However, it is noteworthy that up to half of the respondents did not receive information regarding the causes of their illnesses, and only a few, 1 in 5 mentioned that they had dialogue with health care providers on medication to take. Dialogue is critical towards improving patient compliance and treatment outcomes. It is of paramount importance for health providers to discuss with patients the management of their conditions.

Few health care providers, 4 in 10, were found to be always available in the health facilities. Staff absenteeism in the health facilities negatively impacts on the quality of services especially when the senior trained staff members abdicate their responsibilities to junior staff usually nursing assistants. Several reasons for staff absenteeism have been reported including attending workshops, working in their gardens and fatigue because of being few staff members in a health facility. Studies by Lehman et al. 2008 have highlighted several factors that influence staff decision to accept and stay in remote posts, and strategy by government to respond to such factors that include low wages and poor working conditions [15]. There is need to further explore the reasons for staff ab- 
senteeism in the setting so as to put in place measures to address them. Furthermore, there is need to ensure supportive supervision mechanisms as well as improvement in staff remuneration and motivation so as to minimize staff absenteeism and enhance staff performance.

This study showed that although only a quarter of the health facilities always had drugs available, more than half of the drugs prescribed were available. In the setting, ensuring regular availability of drugs and supplies may be attributable to the collaborative efforts of key stakeholders especially humanitarian United Nations organizations including UNHCR, WHO and international non governmental organization such as IRC that work in emergencies and central and local governments health authorities. The availability of drugs and supplies is vital to the quality of health care services rendered. The support of humanitarian organizations remains critical to the effective operations of local (district) health systems in such emergency settings.

Several barriers to health care accessibility were identified. The main barriers included lack of financial resources, information and decision making power. Poverty is a major factor affecting people's health, access to basic needs, limiting ability to fully participate in society and restricting choices people can make [8]. The internally displaced persons in northern Uganda were in encampment for nearly 2 decades, and subjected to impoverishment, destitution and above all insecurity. Poverty level is highest in the northern war affected districts compared to the other districts in the country [16]. The costs of health care services especially in private not for profit health facilities such as Kalongo hospital deter the majority of the community members from accessing heath care services. Therefore subsidizing of health care services as well as ensuring the displaced populations have ambulance transport services is critical.

Our study showed that most respondents were satisfied with the services provided in the settings. Community satisfaction with health services rendered may be attributable to a variety of reasons including the availability of and accessibility to health related information, and ensuring privacy in the health facilities. However, community members were concerned about the health personnel being rude and uncooperative when providing health services. It is imperative that the reasons underlying staff rudeness are explored and fully comprehended so as to put in place appropriate measures to address them. This is vital towards ensuring improved accessibility, acceptability and quality of health care services in the settings.

\section{CONCLUSION AND RECOMMENDATIONS}

The study suggests that most of the displaced populations lived in close proximity to health facility.
Although geographic access to health services is often high during encampment, this is expected to drastically reduce when the population is resettled in their former homesteads. This suggests the need to establish effective mechanisms for health services delivery in the post conflict period (resettlement phase) including outreach services, strengthening referral system and establishing new first line health facilities. Capacities of first line health facilities including health centre levels (II, IIIs and HCIV) ought to be strengthened. Referral services to higher level care including medical, surgical and emergency obstetric care in the district hospitals should be improved through the availability of ambulance services and other logistical measures including radio or telecommunication networks so as to improve access to health care services.

\section{ACKNOWLEDGEMENTS}

The authors are grateful to WHO staff in Kampala including Dr Olu Olusayho, Nasan Natseri and Annelie Rostedt for the support with the development of the study protocol and data analysis. Similarly we thank Dr Francis Oriokot and Mr David Opio of Kitgum WHO suboffice, Mr Innocent Komakech and Mr Fred Olobo of WHO field office in Pader district for their help with logistical arrangements towards data collection.

\section{REFERENCES}

[1] Office of the Prime Minister (OPM) (2005) Northern Uganda internally displaced persons profiling study. Department of Disaster Preparedness and Refugees.

[2] Office of the Prime Minister (OPM) (2004) The National Policy for internally displaced persons. Department of Disaster Preparedness and Refugees.

[3] Ministry of Health (MoH) (2005) Health and mortality survey among internally displaced persons in Gulu, Kitgum and Pader districts. MoH/WHO Study Report, Northern Uganda.

[4] MSF (1997) Refugee health. An approach to emergency situations. Macmillan Press, London.

[5] Tambiah, Y. (2004) Sexuality and women's right in armed conflicts in Sri lanka. Sexuality, rights and social justice. Reproductive Health Matters, 12, 78-87. doi:10.1016/S0968-8080(04)23121-4

[6] Toole, M.J. and Waldman, R.J. (1993) Refugees and displaced persons. War, hunger and public health. JAMA, 270, 600-605. doi:10.1001/jama.1993.03510050066029

[7] World Health Organization (WHO) (2003) International migration, health and human rights. WHO Publication series No. 4.

[8] Gregorie and Roufail (2005) Racialised groups and health status: A literature review exploring poverty, housing, race-based discrimination and access to health care as determinants of health for racialised group. Access Alliance.

[9] Reitz, J.G. (1995) Review of literature on aspects of ethno-racial access, utilization and delivery of social services. 
Toronto: Multicultural coalition for access to family services and the Ontario Ministry of Community and Social Services. http://ceris.metropolis.net/frametset_e.html

[10] Office for Coordination of Humanitarian Affairs (OCHA) (2007) Fact Sheet No 27. Seventeen frequently asked questions about United Nations special rapporteur. http://www.ohchr.org

[11] World Health Organisation (WHO) (1988) Coverage Survey. WHO Expanded Programme on Immunisation. Training for Mid-level Managers 1988. WHO, Geneva. (WHO/ $\mathrm{EPI} / \mathrm{MLM} / \mathrm{COV} / 88$ ).

[12] Orach, G.C. and De Brouwere, V. (2006) Post emergency health services for refugees and host populations in Uganda, 1999-2002. Lancet, 364, 611-112. doi:10.1016/S0140-6736(04)16854-2
[13] Ministry of Health (MoH) (2009) National Health Policy II. Government of Uganda. Reducing poverty through promoting people's health. Ministy of Health, Kampala, Uganda.

[14] World Health Organisation (WHO) (2002) 25 questions and answers on health and human rights. WHO Health and Human Rights Publication Series Issue No. 1.

[15] Lehman, U., Dieleman, M. and Martineau, T. (2008) Staffing remote rural areas in middle-and low income countries. A literature review of attraction and retention. BMC Health Services Research, 8, 19. doi:10.1186/1472-6963-8-19

[16] United Nations Development Programme (UNDP) (1998) Uganda Human Development Report. Poverty and $\mathrm{Hu}-$ man Development: Kampala Report, 7-26. 